\title{
Effects of detraining in age-group swimmers performance, energetics and kinematics
}

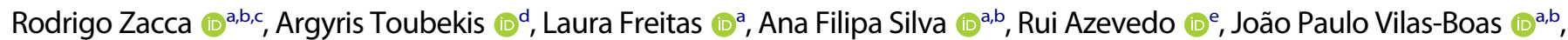 \\ David B. Pyne (1), Flávio A. De S. Castro (1) ${ }^{g}$ and Ricardo J. Fernandes (10) \\ ${ }^{a}$ Centre of Research, Education, Innovation and Intervention in Sport, Faculty of Sport, University of Porto, Porto, Portugal; bPorto Biomechanics \\ Laboratory, University of Porto, Porto, Portugal; 'CAPES Foundation, Ministry of Education of Brazil, Brasília, Brazil; ${ }^{\mathrm{d} N a t i o n a l}$ and Kapodistrian

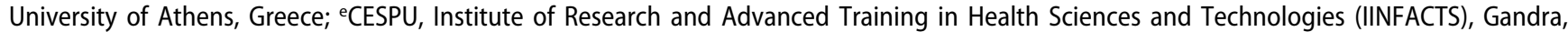 \\ Portugal; fResearch Institute for Sport and Exercise, University of Canberra, Canberra, Australia; ${ }^{9}$ School of Physical Education, Physiotherapy and \\ Dance, Aquatic Sports Research Group, Universidade Federal do Rio Grande do Sul, Brazil
}

ABSTRACT

Changes in performance, energetics and kinematics during age-group swimmers off-season inform the prescription of training for the following season. Age-group swimmers ( $n=15$, age $14.3 \pm 0.7$ years) of equal maturational stage performed a 400-m front crawl (T400) before and after a four-weeks training cessation period. Performance-related energetic and kinematic variables were obtained controlling for anthropometric changes and non-swimming specific physical activities during off-season. T400 time decreased 3.8\% $(95 \% \mathrm{Cl} 1.4$ to $6.1 \% ; \mathrm{p}<0.01 ; d=0.90)$ with non-specific physical activities $\left(1814 \pm 1989 \mathrm{MET}-\mathrm{min}^{\mathrm{w}} \mathrm{wk}^{-1}\right)$ accounting for $\sim 40 \%$ of the underlying variance $\left(p=0.01 ; \eta^{2}=0.40\right)$. Stroke rate and stroke index decreased despite similar stroke length and index of coordination values. Although mean response time, amplitude, maximal oxygen uptake, heart rate, total energy expenditure, metabolic power and energy cost were similar, aerobic contribution decreased by $~ 1.8 \%(-2.7$ to $-0.9 \% ; \mathrm{p}<0.01 ; d=-1.19)$ and anaerobic lactic contribution increased by $\sim 1.6 \%$ ( 0.8 to $2.5 \% ; p<0.01 ; d=1.08$ ) over the off-season. Impaired performance was mainly associated with a decreased stroke rate ( $r=-0.85$ to $-0.61 ; p \leq 0.02)$, increased peak blood lactate $(r=-0.52 ; p=0.05)$ and fewer non-swimming specific physical activities performed during the offseason $(r=-0.58 ; p=0.03)$. The end-of-season cessation of training yielded moderate impairments in agegroup swimmers performance-related energetic and kinematic factors, however non-specific physical activities can minimise fitness losses.
ARTICLE HISTORY

Accepted 14 January 2019

KEYWORDS

Swimming; age-group;

off-season; training

cessation; detraining

\section{Introduction}

Tracking energetic and kinematic changes associated with performance provides important insights into swimmers preparation, but detailed information on parallel changes in swimming performance are difficult to obtain in the aquatic environment (Ayabakan et al., 2006; Csajagi, Szauder, Major, \& Pavlik, 2015; Morais, Marques, Marinho, Silva, \& Barbosa, 2014). Swimming-related studies are mostly cross-sectional in approach and, although relevant, they do not provide conclusive information about cause-and-effect relationships between swimming determinant variables and performance during training and off-season phases. Therefore, longitudinal data on swimmers training is still required.

The partial or complete loss of training-induced anatomical, physiological and functional adaptations with reduced training, or even training cessation, is termed detraining (Mujika \& Padilla, 2000). Detraining can be caused by illness, injury or, more commonly, the post-season break. Typically, a four-weeks off-season is applied for high-level swimmers, a period used in detrainingrelated studies (Mujika \& Padilla, 2000). Age-group swimmers typically recover for several weeks in the off-season, but this duration, usually 4-6 weeks, may differ according to requirements of individual coaches and/or the calendar of each national swimming federation (Costill et al., 1985; Garrido et al., 2010). In contrast to most senior swimmers, young swimmers usually enjoy the offseason with complete cessation of training. Although coaches often report reductions in fitness and loss of technique, evidencebased outcomes on the effects of detraining in young adolescent swimmers are scarce. Likewise, training reduction is important for performance improvements, but the effects of the duration of training cessation and its impact on performance are not well reported for age-group swimmers. It is also unclear the extent to which critical energetic and kinematic characteristics can change during the off-season. Although these breaks in training are planned to allow for some relief from the stresses of training, if prolonged they might produce decrements in both energetic and kinematics performance related variables. Minimising impairments in swimming performance during the transition to the following competitive season is important for technical continuity, since identifying which changes might account for performance impairment can guide coaches in planning the next season's training program (Costill et al., 1985).

Longitudinal studies on physiological and morphological changes in age-group swimmers indicate that performance 
improvements are dependent on growth and training stimuli (Ayabakan et al., 2006; Csajagi et al., 2015; Kavouras \& Troup, 1996). Likewise, sex differences and intra-individual variability for performance-related kinematic factors have been described over two competitive seasons (Barbosa et al., 2015; Morais et al., 2014). However, energetic and kinematic adaptations are transitory, and might decline when training load is reduced after the main competition of the season. This effect could explain why some coaches encourage age-group swimmers to undertake nonswimming specific physical activities (like dry land sports, crosstraining or gym-based exercises) during the off-season. However, the effects of these land physical activities need to be quantified, since they may offset the detraining related to swimming training. For instance, Garrido et al. (2010) indicated that strength remained stable while 25 - and 50-m swimming performance improved after six-weeks of training cessation in 12 years old age-group swimmers, but the effects of growth and non-specific physical activities performed during that period were not controlled.

Performance during a swimming event depends on the conversion of metabolic into mechanical power through a given energetic efficiency (Barbosa et al., 2010; Figueiredo, Pendergast, Vilas-Boas, \& Fernandes, 2013). The total energy expenditure $\left(E_{\text {tot }}\right)$, defined as the sum of aerobic (Aer), anaerobic lactic $(A n L)$ and anaerobic alactic ( $A n A L)$ energy contributions during different events, the metabolic power (E), energy expenditure per unit of time) and the energy cost ( $C$; the energy consumed to cover one unit of distance while swimming at a given speed), have been well studied through indirect calorimetry (Sousa, Figueiredo, Zamparo, Vilas-Boas, \& Fernandes, 2013). Detraining during the off-season may increase the oxygen uptake $\left(\mathrm{VO}_{2}\right)$ and blood lactate concentrations ([ $\left.\left.\mathrm{La}^{-}\right]\right)$for a given effort, making it more difficult to sustain the swimming speeds (v) reached at the end of the previous season. Maximal oxygen uptake $\left(\mathrm{V}_{2 \text { max }}\right)$ is a relevant energetic parameter of aerobic function in swimming, and analysis of the dynamic $\dot{\mathrm{VO}}_{2}$ response following the onset of exercise $\left(\mathrm{V}_{2}\right.$ kinetics) can provide a more detailed picture of its cardiorespiratory determinants (Ribeiro et al., 2017). To date no study has yet quantified the effects of detraining on $\mathrm{VO}_{2}$ kinetics after the off-season in age-group swimmers. It is plausible that this pause in training may negatively influence $\dot{\mathrm{V}}_{2}$ related parameters and the subsequent progression of adolescent swimmers.

Age-group swimmers technique is also influenced by training that underpins improvements in swimming performance over a training season (Morais et al., 2014). Improvements in swimming kinematics after $\sim 10$-weeks of off-season in age-group swimmers were largely explained by anthropometric changes related to growth (Moreira et al., 2014). However, the effects of non-swimming specific physical activity were also not controlled. Thus, growth and non-swimming physical activities effects should be accounted when assessing performance changes through prospective detraining studies, since they could reduce, at least in part, the loss of swimming-specific fitness during the transition to the next competitive season (Neufer, 1989). The aim of the study was to assess the performance, energetic and kinematic effects of a typical off-season period in age-group swimmers. The anthropometric growth and non-swimming specific physical activities performed during this period of swimming training cessation were controlled. We expected that training cessation at the end of a training season would yield impairments in performance, energetics and technical variables, partially offset by a swimmer's growth and non-specific physical activities during the transition period.

\section{Methods}

\section{Experimental approach to the problem}

A longitudinal single cohort study was conducted, and swimmers were tested before and after a four-weeks off-season period. Repeated measures of performance-related energetic and kinematic variables, controlling for anthropometric and nonswimming specific physical activities, were obtained. The first experimental testing (PRE) was conducted until 48 hours after the main competition of the year, remaining only few days left of training (with low volume and training load) until swimmers commenced their four-weeks off-season. Swimmers were then instructed to refrain from swimming-pool training during the whole off-season. The second experimental testing (POST) was conducted four-weeks later, until 48 hours after the start of the first week of the new training season (See Figure 1).

Anthropometric variables and pubertal maturation stage were assessed in both testing sessions, and self-reported physical activity data from the off-season was obtained in the POST evaluation. The experimental protocol took place in a $25-\mathrm{m}$ indoor pool with 27.5 and $25.9^{\circ} \mathrm{C}$ of water and air temperatures (respectively) and $65 \%$ relative humidity. After an 800-m moderate intensity front crawl warm-up, swimmers performed a 400-m maximal effort front crawl swim test (T400) for performance, energetic and kinematic assessment. The T400 was chosen given its validity and feasibility for assessing aerobic power in age-group swimmers (Laffite et al., 2004; Zacca et al., 2017). All participants avoided vigorous exercise in the previous $24 \mathrm{~h}$, were well-hydrated, abstained from caffeine for, at least, $3 \mathrm{~h}$ before testing and encouraged verbally during the $\mathrm{T} 400$.

\section{Participants}

Fifteen late to post pubertal age-group swimmers, six boys and nine girls (14.5 \pm 0.8 and $14.2 \pm 0.6$ years, respectively), volunteered to participate in the current study. Swimmers had over 5 years of competitive experience and trained six to seven swimming sessions per week in the same squad and under direction of the same coach. Swimmers parents were informed about the benefits and risks of participating in the current study prior to signing an informed consent form, which was approved by the ethics board of the local university in accordance with the Helsinki Declaration.

\section{Procedures}

Standard methodologies were used to compute the swimming training workload (Mujika et al., 1996). The transition period (offseason) coincided with the school holidays (Tschiene, 1977). The swimming season involved a traditional three-peak preparation program (Seiler, 2010; Tschiene, 1977). Swimming training prior 


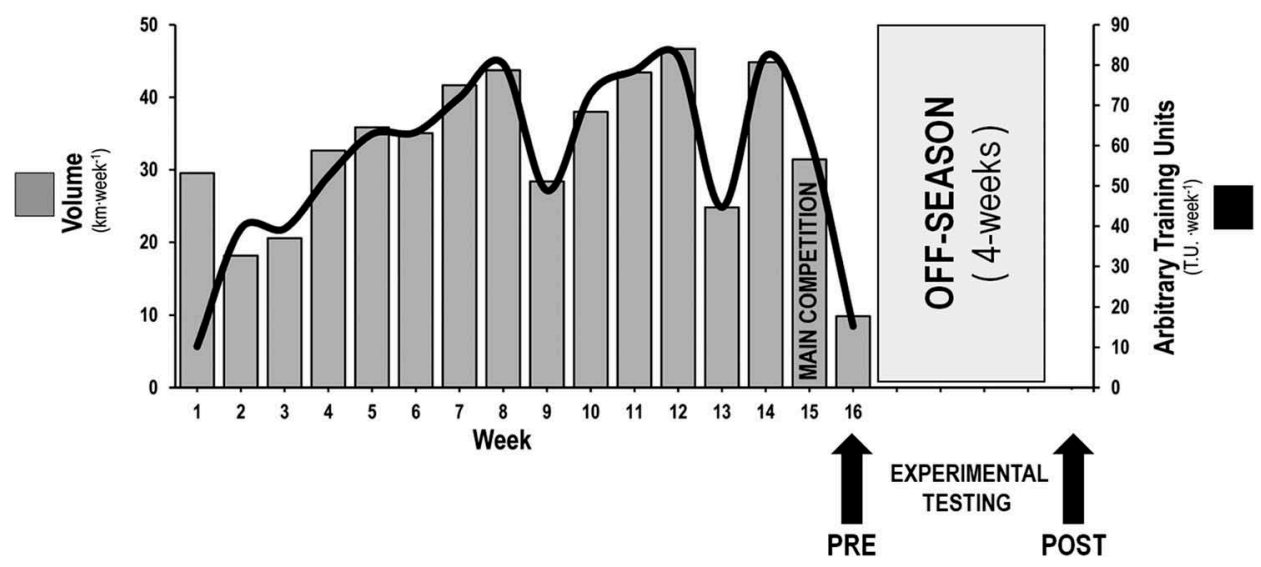

Figure 1. Training volume and units (T.U.) over the last 16-weeks macrocycle immediately before the off-season.

to the off-season was categorised using a five-training zone system (Mujika et al., 1995), performed by the swimmers from the same squad and under direction of the same coach. Swimming distances (volume) were multiplied by the intensity factor and totalled (Mujika et al., 1996). The training load was expressed in arbitrary training units (T.U.), which was quantified from the sum of the volumes swum in each of the five training zones, multiplied by the respective intensity factor, and the total volume completed (Mujika et al., 1996, 1995; Tschiene, 1977). Thus, swimmers' volume and T.U. were quantified over 16 weeks, and the progression of training was monitored through the last macrocycle of the season (Hellard, Avalos, Guimaraes, Toussaint, \& Pyne, 2015; Vanrenterghem, Nedergaard, Robinson, \& Drust, 2017; Walsh et al., 2011). Figure 1 summarises the training volume and load (T.U.) over the last 16-weeks macrocycle immediately before the off-season.

Anthropometric profile (body mass, height, arm span and body mass index) was obtained by an International Society for the Advancement of Kinanthropometry accredited level I anthropometrist. Maturational status was verified by a valid and reliable self-assessment of secondary sexual characteristics to determine the degree of homogeneity of the subject cohort of this study (Tanner \& Whitehouse, 1976). Total physical activity was assessed by a weekly self-reported short questionnaire (International Physical Activity Questionnaire - IPAQ; Craig et al., 2003) and summarised according to the physical activities recorded (low, moderate and vigorous activities). The results of the swimmers' questionnaires were converted into units of metabolic equivalent of task (METs) following the IPAQ specifications. Swimmers were advised to record their daily activities for their own control. The IPAQ has adequate test-retest reliability with a mean correlation of $\sim 0.80$ ranging from "fair" 0.46 to "excellent" 0.96 (Craig et al., 2003)

Respiratory and pulmonary gas-exchange data were measured breath-by-breath using a telemetric portable gas analyzer (K4b², Cosmed, Rome, Italy) connected to a low hydrodynamic resistance respiratory snorkel and valve system (AquaTrainer ${ }^{\circledast}$, Cosmed, Rome, Italy; Ribeiro et al., 2016). The telemetric portable gas analyser was calibrated before each testing session and transported on a steel cable along the swimming pool suspended at a 2-m height over the water (de Jesus et al., 2014). Swimmers were familiarised during three months before the PRE testing, three times per week, with a snorkel (Arena swim snorkel SKU: 95,257; Tolentino, Italy) and nose clip (Arena nose clip pro SKU: 95,204; Tolentino, Italy). In-water starts and open turns were employed given the physical restrictions associated with the equipment (Ribeiro et al., 2016). Heart rate (HR) was monitored continuously by a Polar Vantage NV (Polar Electro Oy, Kempele, Finland) that transmitted the data telemetrically to the $\mathrm{K}_{4} \mathrm{~b}^{2}$ portable unit. Capillary blood samples for $\left[\mathrm{La}^{-}\right]$were collected from an earlobe before exercise and immediately after the T400 at the first, third, fifth and seventh min of the recovery period ([ $\left.\mathrm{La}^{-}\right]_{\text {peaki }}$ Lactate Pro analyzer, Arkray, Inc, Kyoto, Japan).

The first $20 \mathrm{~s}$ of $\dot{\mathrm{VO}}_{2}$ data after the onset of exercise (the cardiodynamic phase of the $\dot{\mathrm{V}}_{2}$ response) were not considered for $\dot{\mathrm{V}}_{2}$ kinetics analysis and errant breaths were omitted by only including those within $\mathrm{VO}_{2}$ local mean $\pm 4 \mathrm{SD}$ (Sousa, Vilas-Boas, \& Fernandes, 2014). Subsequently, individual breath-by-breath $\mathrm{V}_{2}$ responses were time averaged every $10 \mathrm{~s}$ and smoothed using a 3-breath moving average (de Jesus et al., 2014). The on-transient $\dot{\mathrm{VO}}_{2}$ responses during the T400 were modelled for each swimmer using the mono exponential model described in Equation (1):

$$
\dot{\mathrm{V}} \mathrm{O}_{2}(\mathrm{t})=\mathrm{A}_{0}+\mathrm{H}\left(\mathrm{t}-\mathrm{TD}_{\mathrm{p}}\right) \cdot \mathrm{A}_{\mathrm{p}}\left(1-\mathrm{e}^{-\left(\mathrm{t}-\mathrm{TD}_{\mathrm{p}}\right) / \tau_{\mathrm{p}}}\right)
$$

where $\dot{\mathrm{V}}_{2}(t)$ represents the relative $\dot{\mathrm{VO}}_{2}$ at the time $t, \mathrm{~A}_{0}$ is the $\mathrm{V}_{2}$ at rest (average from the last 2 min previous to exercise), $\mathrm{H}$ represents the Heaviside step function (Equation (2); $\mathrm{Ma}$, Rossiter, Barstow, Casaburi, \& Porszasz, 2010) and $A_{p}, T_{p}$ and $\tau_{p}$ are the amplitude, time delay and time constant of the fast $\dot{\mathrm{VO}}_{2}$ component.

$$
\mathrm{H}(\mathrm{t})=\left\{\begin{array}{l}
0, t<0 \\
1, t \geq 0
\end{array}\right.
$$

Since T400 performances were different between swimmers, mean $\mathrm{VO}_{2}$ over the last $30 \mathrm{~s}$ of each $100-\mathrm{m}$ interval was calculated to characterise and compare $\mathrm{T} 400 \mathrm{~V}_{2}$ responses (Hanon, Leveque, Thomas, \& Vivier, 2008; Ingham, Fudge, Pringle, \& Jones, 2013; Zacca et al., 2017). The mean response time (MRT) was obtained as $\mathrm{TD}_{\mathrm{p}}+\tau_{\mathrm{p}}$ and $\dot{\mathrm{VO}}_{2 \max }$ computed as 
the average of the last $30 \mathrm{~s} \dot{\mathrm{VO}}_{2}$ values during the T400. It is well-established that swimmers can reach and sustain the minimal $v$ that elicits $\dot{\mathrm{V}}_{2 \max }\left(\mathrm{v}_{\mathrm{V}} \mathrm{O}_{2 \max }\right)$ during a $400-\mathrm{m}$ effort (Billat et al., 1996; Fernandes et al., 2006; Zacca et al., 2017).

The estimated $E_{\text {tot }}$ was calculated as the sum of Aer, AnL and $A n A L$ energy contributions, with the Aer and AnL energy calculated from the time integral of the net $\dot{\mathrm{VO}}_{2}$ versus time relationship and using the Equation (3), respectively (Sousa et al., 2014):

$$
\mathrm{AnL}=\left[\mathrm{La}^{-}\right]_{\text {net }} \cdot \beta \cdot \mathrm{M}
$$

where $\left[\mathrm{La}^{-}\right]_{\text {net }}$ is the difference between the $\left[\mathrm{La}^{-}\right]$before and after exercise $\left(\left[\mathrm{La}^{-}\right]_{\text {peak }}\right), \beta$ is the constant for $\mathrm{O}_{2}$ equivalent of $\left[\mathrm{La}^{-}\right]_{\text {net }}\left(2.7 \mathrm{ml} \cdot \mathrm{kg}^{-1} \cdot \mathrm{mM}^{-1}\right.$; Thevelein, Daly, \& Persyn, 1984) and $M$ is the body mass of the swimmer. These energy contributions were then expressed in $\mathrm{kJ}$ assuming an energy equivalent of $20.9 \mathrm{~kJ} \cdot \mathrm{L}^{-1}$ (Zamparo, Capelli, \& Pendergast, 2011). The AnAL energy was estimated from the maximal phosphocreatine $(\mathrm{PCr})$ splitting in the contracting muscle (Equation (4); Sousa et al., 2014; Binzoni, Ferretti, Schenker, \& Cerretelli, 1992):

$$
A n A L=P C r \cdot\left(1-e^{-t / \tau}\right) \cdot M
$$

where $\mathrm{PCr}$ is the phosphocreatine concentration at rest, $\mathrm{t}$ is the exercise time, $\tau$ is time constant of the $\mathrm{PCr}$ splitting at exercise onset (23.4 s) and $\mathrm{M}$ is the body mass. Subsequently, AnAL was expressed in $\mathrm{kJ}$ by assuming an energy equivalent of $0.468 \mathrm{~kJ} \cdot \mathrm{mM}^{-1}$ and a phosphate/oxygen ratio of 6.25 (Zamparo et al., 2011). The $C$ was obtained as the ratio between $E_{\text {tot }}$ and distance, and metabolic power ( $\dot{E}_{;} k W$ ) was estimated as the ratio between $E_{\text {tot }}$ and performance (s) of T400 (Zamparo et al., 2011).

Surface and underwater video cameras $\left(50 \mathrm{~Hz}\right.$, Sony ${ }^{\circledR}$ Handycam HDR-CX130, Japan) recorded $10-\mathrm{m}$ of the last $25-\mathrm{m}$ of each $100-\mathrm{m}$ interval (within two points at $7.5 \mathrm{~m}$ from each end of the swimming-pool to exclude the influence of the turning phase). Cameras were synchronised with Adobe Premiere Pro CC (v2015, Adobe Systems, San Jose, California, USA) through a light flash activated prior to each trial. The $v$ from each $100-\mathrm{m}$ interval was measured from the time taken to cover the middle $10-\mathrm{m}$ of each length $(v=d /$ $\mathrm{t} 10$, where $\mathrm{d}=10-\mathrm{m}$ and $\mathrm{t} 10=$ time for the $10 \mathrm{~m}$ ) and the $\mathrm{v}$ of T400 was calculated as the mean of the four 100-m intervals. Stroke rate (SR) was computed from the time taken to complete three consecutive upper limb cycles and then converted in cycles per minute. Stroke length (SL) was calculated from the ratio between $v$ and corresponding SR. Stroke index (SI), a measure of swimming efficiency, calculated by multiplying $v$ by SL (Costill et al., 1985). Inter-limb coordination was assessed by the Index of Coordination (IdC; Chollet, Chalies, \& Chatard, 2000; Figueiredo, Toussaint, VilasBoas, \& Fernandes, 2013; Seifert et al., 2014) where the lag time between the propulsive phases of each upper limb (two cycles) was expressed as a percentage of the overall duration of the upper limbs cycle. Following this index, arm propulsive phase begins with the start of the hand's backward movement and ends when it exits from the water (pull and push phases). The non-propulsive phase starts when the hand releases from the water, ending at the beginning of the propulsive phase (recovery, entry, and catch phases). The three coordination modes attributed to front crawl swimming are "catch-up", when a lag time occurs between the propulsive phases of both arms (IdC < 0\%); "opposition", when the propulsive phase of one arm starts when the other arm ends its propulsive phase (IdC =0\%); and "superposition", when the propulsive phases of both arms overlap (IdC > 0\%) (Chollet et al., 2000).

\section{Statistical analysis}

A longitudinal cohort study analysis with PRE and POST repeated measures (performance, energetic and kinematic related variables, anthropometric and non-swimming physical activity) was performed. Normality and homogeneity were verified and confirmed with the Shapiro-Wilk's and Levene's Tests respectively. Mean and SD for descriptive analysis were obtained and reported for all studied variables. A Fisher's test was used to test for differences in pubertal stage between male and female swimmers. Possible sex effects (covariate) between PRE and POST paired means for all variables were verified a priori. Bootstraping with 1000 samples was used to estimate $\dot{\mathrm{V}}_{2}$ kinetics parameters and respective coefficient of variation. A paired t-test was used to compare differences between PRE and POST off-season for each variable. Effect sizes (Cohen's $d$ ) were interpreted with the following criteria: 0-0.19 trivial, 0.2-0.59 small, 0.6-1.19 moderate, 1.2-1.99 large, 2.0-3.99 very large and $>4.0$ nearly perfect (Hopkins, 2002).

Partial correlations, conditioning on sex, were used to quantify the degree of association between deltas $(\Delta$, i.e., POST values subtracted by PRE values) for each variable and the change in T400 performance, interpreted as follows: $<0.40$ poor, $0.40-0.75$ fair to good and $>0.75$ excellent (Fleiss, 1986). Repeated measures analysis of variance (ANOVA) was performed with PRE and POST performances using the change score values of height, body mass and arm span, and the total physical activity during off-season as a covariate to control the effects of anthropometric changes and non-swimming specific physical activities. Eta squared $\left(\eta^{2}\right)$ test was used to quantify the percentage of the variance explained by each covariate (effect size) and interpreted as follows: $0<\eta^{2}<0.04$ without effect, $0.04 \leq \eta^{2} \leq 0.24$ minimum, $0.25 \leq \eta^{2}<0.64$ moderate and $\eta^{2} \geq 0.64$ large effect (Ferguson, 2009). Alpha significance level was established at 0.05 .

\section{Results}

No differences $(p=0.455)$ in pubertal maturation stage were identified between male and female swimmers. On the basis of trivial sex effects ( $p=0.06-0.97 ; \eta^{2}=0.00$ to 0.23 ) observed for all variables during preliminary data analysis, male and female swimmers were pooled and analysed as a single group. The mean volume and training load per week over the last 16-week macrocycle immediately before the offseason were $33 \pm 11 \mathrm{~km} \cdot$ week $^{-1}$ and $57 \pm 23 \mathrm{~T}$.U. $\cdot$ week $^{-1}$ respectively. Progression of volume and training load until two weeks prior to the main competition of this macrocycle were $\sim 12$ and $\sim 10 \% \cdot$ week $^{-1}$ respectively (Figure 1 ). 
Height, arm span and weight values were higher at the beginning of the new training season than in the final of the previous one ( $p<0.05 ; d \geq 0.90$ ) but had little influence on T400 performance changes (Table 1). The body mass index was similar between PRE and POST ( $p=0.33$; mean difference: $0.2 ; 95 \% \mathrm{Cl}:-0.20$ to $0.56 ; d=0.25$ ). However, reductions in swimming performance were attenuated by completion of non-swimming specific physical activities during the offseason $\left(1814 \pm 1989\right.$ MET-min.wk $\left.{ }^{-1}\right)$, as they accounted for $40 \%$ of the total variance in performance $(p=0.01$; partial $\eta^{2}=0.40$ moderate) and showed good partial correlation with the reduction in T400 performance ( $r=-0.58$; $p=0.03)$. Low intensity non-swimming specific physical activities during the off-season, isolated, did not account for much of the total variance in performance $\left(p=0.13\right.$; partial $\left.\eta^{2}=0.16\right)$. Moderate intensity non-swimming specific physical activities during the off-season, accounted for $31 \%$ of the total variance in performance ( $p=0.03$; partial $\eta^{2}=0.31$ moderate) but partial correlation with the reduction in T400 performance was not significant. Vigorous intensity non-swimming specific physical activities during the off-season, accounted for $42 \%$ of the total variance in performance $\left(p=0.009\right.$; partial $\eta^{2}=0.42$ moderate) and showed good partial correlation with the reduction in T400 performance $(r=-0.61 ; p=0.021)$.

T400 swimming performance was impaired by 3.8\% (1.4-6.1\%; mean $(95 \% \mathrm{Cl}) ; \mathrm{p}<0.01 ; d=0.90)$ during the off-season, with the $\mathrm{v}$ being markedly slower at 100-, 200-, 300- and 400-m laps ( $p \leq 0.01 ; d=-5.20$ to -1.06 ; moderate to very large effect) at the POST condition (Table 2). Likewise, SR decreased at all 100-m intervals ( $p \leq 0.01 ; d=-1.27$ to -0.77 ), which influenced SI values (also substantially lower at all 100-m intervals; $\mathrm{p}<0.05 ; d=-0.94$ to -0.66$)$ in the beginning of the new training season. Both SL ( $p=0.36-0.74 ; d=-0.25$ to 0.09$)$ and IdC ( $p=0.19-0.89 ; d=-0.36$ to -0.04 ) were similar between PRE and POST at all 100-m intervals. There was good to excellent inverse associations between changes in SR and T400, with a lower SR at all 100-m intervals at

Table 1. Effects of four-weeks off-season on age-group swimmers anthropometrics. Non-swimming specific physical activities engaged by swimmers during the offseason and Tanner stage are also shown. There are displayed the PRE and POST mean \pm SD values with respective level of probabilities ( $p$ ), mean differences, 95\% confidence intervals, relative changes $(\% \Delta)$, significant effect sizes and partial correlations between deltas and delta performance $(\Delta)$.

\begin{tabular}{|c|c|c|c|c|c|c|}
\hline Variable & PRE & POST & $\mathrm{p}$ & Difference $[95 \% \mathrm{Cl}] ; \% \Delta$ & Effect size $(d)$ & $\begin{array}{c}\Delta \text { vs } \Delta \text { Performance } \\
\text { Partial Correlation }(p)\end{array}$ \\
\hline Height (cm) & $164.3 \pm 6.2$ & $165.7 \pm 5.8$ & $<0.01$ & $1.4[0.7,2.4] ; 0.9 \%$ & 1.05, moderate & $0.30(0.31)$ \\
\hline Arm span $(\mathrm{cm})$ & $167.5 \pm 8.3$ & $168.7 \pm 8.8$ & $<0.01$ & $1.2[0.5,1.9] ; 0.7 \%$ & 0.98 , moderate & $0.40(0.16)$ \\
\hline Body mass $(\mathrm{kg})$ & $59.6 \pm 10.3$ & $61.1 \pm 10.5$ & $<0.01$ & $1.5[0.6,2.4] ; 2.5 \%$ & 0.98 , moderate & $-0.34(0.23)$ \\
\hline Body mass index $\left(\mathrm{kg} \cdot \mathrm{m}^{2}\right)$ & $21.9 \pm 2.7$ & $22.1 \pm 2.6$ & - & - & - & $-0.43(0.13)$ \\
\hline Low intensity $\left(\right.$ MET-min $\cdot w^{-1}$ ) & - & $485 \pm 643$ & - & - & - & $-0.39(0.16)$ \\
\hline Moderate intensity $\left(\mathrm{MET}-\mathrm{min} \cdot \mathrm{wk}^{-1}\right)$ & - & $935 \pm 1013$ & - & - & - & $-0.49(0.08)$ \\
\hline Vigorous intensity $\left(\mathrm{MET}-\mathrm{min} \cdot \mathrm{wk}^{-1}\right)^{\prime}$ & - & $394 \pm 652$ & - & - & - & $-0.61(0.02)$ \\
\hline Total physical activity $\left(\right.$ MET-min $\cdot$ wk $^{-1}$ ) & - & $1814 \pm 1989$ & - & - & - & $-0.58(0.03)$ \\
\hline Tanner stage * $(\mathrm{I} / \mathrm{II} / \mathrm{III} / \mathrm{IV} / \mathrm{V})$ & $\begin{array}{l}\text { Q }(0 / 0 / 0 / 6 / 3) \\
\sigma^{n}(0 / 0 / 0 / 3 / 3)\end{array}$ & - & - & - & - & - \\
\hline
\end{tabular}

-: Absence of values for PRE and POST comparison or Not significantly different.

*Tanner: prepubertal (Tanner stage I), early-pubertal (Tanner stage II,), peripubertal (Tanner III), latepubertal (Tanner IV) and postpubertal (Tanner V). o female.

o' male

Table 2. Effects of four-weeks off-season on age-group swimmers kinematics. There are displayed the PRE and POST mean \pm SD values with respective level of probabilities $(p)$, mean differences, $95 \%$ confidence intervals, relative changes $(\% \Delta)$, significant effect sizes and partial correlations between deltas and delta performance $(\Delta)$.

\begin{tabular}{|c|c|c|c|c|c|c|}
\hline Variable & PRE & POST & $\mathrm{p}$ & Difference $[95 \% \mathrm{Cl}] ; \% \Delta$ & Effect size $(d)$ & $\begin{array}{c}\Delta \text { vs } \Delta_{\text {Performance }} \\
\text { partial correlation }(p)\end{array}$ \\
\hline Performance (s) & $345 \pm 20$ & $358 \pm 21$ & $<0.01$ & $13[5,21] ; 3.8 \%$ & 0.90 , moderate & $-(-)$ \\
\hline $\mathrm{v} 100\left(\mathrm{~m} \cdot \mathrm{s}^{-1}\right)$ & $1.14 \pm 0.06$ & $1.08 \pm 0.07$ & $<0.01$ & $-0.06[-0.09,-0.03] ;-5.3 \%$ & -1.20, Large & $-0.45(0.11)$ \\
\hline $\mathrm{v} 200\left(\mathrm{~m} \cdot \mathrm{s}^{-1}\right)$ & $1.13 \pm 0.07$ & $1.06 \pm 0.07$ & $<0.01$ & $-0.07[-0.11,-0.04] ;-6.2 \%$ & -1.06, Moderate & $-0.59(0.03)$ \\
\hline v300 $\left(\mathrm{m} \cdot \mathrm{s}^{-1}\right)$ & $1.14 \pm 0.07$ & $1.06 \pm 0.06$ & 0.01 & $-0.08[-0.13,-0.04] ;-7.4 \%$ & -1.12 , Moderate & $-0.77(<0.01)$ \\
\hline $\mathrm{v} 400\left(\mathrm{~m} \cdot \mathrm{s}^{-1}\right)$ & $1.21 \pm 0.09$ & $1.11 \pm 0.07$ & $<0.01$ & $-0.10[-0.15,-0.06] ;-8.6 \%$ & -5.20, Nearly Perfect & $-0.68(0.01)$ \\
\hline$v\left(m \cdot s^{-1}\right)$ & $1.16 \pm 0.06$ & $1.08 \pm 0.06$ & $<0.01$ & $-0.08[-0.11,-0.05] ;-6.9 \%$ & -1.40, Large & $-0.73(<0.01)$ \\
\hline $\mathrm{SR}_{100}\left(\right.$ cycles $\cdot \mathrm{min}^{-1}$ ) & $33.48 \pm 2.33$ & $31.54 \pm 2.50$ & $<0.01$ & $-1.94[-3.32,-0.57] ;-5.8 \%$ & -0.78, Moderate & $-0.71(<0.01)$ \\
\hline $\mathrm{SR}_{200}\left(\right.$ cycles $\cdot \mathrm{min}^{-1}$ ) & $33.51 \pm 2.67$ & $31.54 \pm 2.72$ & $<0.01$ & $-1.96[-3.32,-0.61] ;-5.9 \%$ & -0.81, Moderate & $-0.77(<0.01)$ \\
\hline $\mathrm{SR}_{300}\left(\right.$ cycles $\left.\cdot \mathrm{min}^{-1}\right)$ & $33.91 \pm 3.19$ & $31.82 \pm 2.65$ & 0.01 & $-2.09[-3.59,-0.59] ;-6.2 \%$ & -0.77, Moderate & $-0.85(<0.01)$ \\
\hline $\mathrm{SR}_{400}\left(\right.$ cycles $\cdot \mathrm{min}^{-1}$ ) & $35.61 \pm 2.96$ & $32.76 \pm 2.62$ & $<0.01$ & $-2.85[-4.09,-1.61] ;-8.0 \%$ & -1.27, Large & $-0.61(0.02)$ \\
\hline $\mathrm{SL}_{100}(\mathrm{~m})$ & $2.06 \pm 0.18$ & $2.07 \pm 0.19$ & - & - & - & $0.60(0.02)$ \\
\hline $\mathrm{SL}_{200}(\mathrm{~m})$ & $2.04 \pm 0.19$ & $2.03 \pm 0.22$ & - & - & - & $0.44(0.12)$ \\
\hline $\mathrm{SL}_{300}(\mathrm{~m})$ & $2.04 \pm 0.19$ & $2.01 \pm 0.18$ & - & - & - & $0.37(0.19)$ \\
\hline $\mathrm{SL}_{400}(\mathrm{~m})$ & $2.06 \pm 0.19$ & $2.04 \pm 0.19$ & - & - & - & $0.03(0.92)$ \\
\hline $\mathrm{SI}_{100}\left(\mathrm{~m}^{2} \cdot \mathrm{s}^{-1}\right)$ & $2.4 \pm 0.3$ & $2.2 \pm 0.3$ & 0.02 & $-0.11[-0.21,-0.02] ;-4.8 \%$ & -0.66, Moderate & $0.11(0.70)$ \\
\hline $\mathrm{SI}_{200}\left(\mathrm{~m}^{2} \cdot \mathrm{s}^{-1}\right)$ & $2.3 \pm 0.3$ & $2.1 \pm 0.3$ & 0.01 & $-0.16[-0.28,-0.04] ;-7.0 \%$ & -0.76, Moderate & $-0.10(0.73)$ \\
\hline $\mathrm{SI}_{300}\left(\mathrm{~m}^{2} \cdot \mathrm{s}^{-1}\right)$ & $2.3 \pm 0.3$ & $2.1 \pm 0.3$ & $<0.01$ & $-0.20[-0.32,-0.08] ;-8.6 \%$ & -0.94, Moderate & $-0.31(0.28)$ \\
\hline $\mathrm{SI}_{400}\left(\mathrm{~m}^{2} \cdot \mathrm{s}^{-1}\right)$ & $2.5 \pm 0.4$ & $2.3 \pm 0.3$ & $<0.01$ & $-0.24[-0.39$ to -0.08$] ;-9.4 \%$ & -0.83, Moderate & $-0.45(0.11)$ \\
\hline $\mathrm{IdC}_{100}(\%)$ & $-9.3 \pm 3.5$ & $-10.1 \pm 3.3$ & - & - & - & $-0.11(0.70)$ \\
\hline $\mathrm{IdC}_{200}(\%)$ & $-9.3 \pm 4.2$ & $-9.7 \pm 3.7$ & - & - & - & $0.47(0.09)$ \\
\hline $\mathrm{IdC}_{300}(\%)$ & $-9.5 \pm 5.0$ & $-9.7 \pm 3.2$ & - & - & - & $0.30(0.30)$ \\
\hline $\mathrm{IdC}_{400}(\%)$ & $-8.5 \pm 4.1$ & $-9.7 \pm 3.8$ & - & - & - & $0.44(0.12)$ \\
\hline
\end{tabular}

v: swimming speed; SR: stroke rate; SL: stroke length; SI: stroke index; IdC: index of coordination; -: Not significantly different. 
Table 3. Effects of four-weeks off-season on age-group swimmers energetics. There are displayed the PRE and POST mean \pm SD values with respective level of probabilities $(p)$, mean differences, $95 \%$ confidence intervals, relative changes $(\% \Delta)$, significant effect sizes and partial correlations between deltas and delta performance $(\Delta)$.

\begin{tabular}{|c|c|c|c|c|c|c|}
\hline Variable & PRE & POST & $\mathrm{p}$ & Difference $[95 \% \mathrm{Cl}] ; \% \Delta$ & Effect size $(d)$ & $\begin{array}{c}\Delta \text { vs } \Delta \text { Performance } \\
\text { partial correlation }(p)\end{array}$ \\
\hline MRT (s) & $43.2 \pm 10.3$ & $46.8 \pm 17.1$ & - & - & - & $0.34(0.24)$ \\
\hline$A_{p}\left(\mathrm{~mL} \cdot \mathrm{kg}^{-1} \cdot \mathrm{min}^{-1}\right)$ & $36.4 \pm 6.0$ & $32.9 \pm 6.4$ & - & - & - & $-0.17(0.55)$ \\
\hline$\dot{\mathrm{V}} \mathrm{O}_{2} 100\left(\mathrm{~mL} \cdot \mathrm{kg}^{-1} \cdot \mathrm{min}^{-1}\right)$ & $44.4 \pm 4.6$ & $38.5 \pm 5.7$ & $<0.01$ & $-5.9[-8.5,-3.3] ;-13.3 \%$ & -1.26, Large & $-0.20(0.48)$ \\
\hline$\dot{\mathrm{V}} \mathrm{O}_{2} 200\left(\mathrm{~mL} \cdot \mathrm{kg}^{-1} \cdot \mathrm{min}^{-1}\right)$ & $44.2 \pm 6.0$ & $39.3 \pm 6.1$ & 0.02 & $-4.9[-9.1,-0.8] ;-11.2 \%$ & -0.66, Moderate & $-0.29(0.31)$ \\
\hline$\dot{\mathrm{V}} \mathrm{O}_{2} 300\left(\mathrm{~mL} \cdot \mathrm{kg}^{-1} \cdot \mathrm{min}^{-1}\right)$ & $44.0 \pm 5.9$ & $40.1 \pm 6.2$ & - & - & - & $-0.38(0.18)$ \\
\hline$\dot{\mathrm{VO}}_{2} 400\left(\dot{\mathrm{VO}}_{2 \mathrm{max}}\right)\left(\mathrm{mL} \cdot \mathrm{kg}^{-1} \cdot \mathrm{min}^{-1}\right)$ & $45.4 \pm 5.7$ & $42.3 \pm 6.6$ & - & - & - & $-0.31(0.27)$ \\
\hline$\dot{\mathrm{VO}}_{2} 400\left(\dot{\mathrm{VO}}_{2 \mathrm{max}}\right)\left(\mathrm{L} \cdot \mathrm{min}^{-1}\right)$ & $2.68 \pm 0.47$ & $2.58 \pm 0.6$ & - & - & - & $-0.48(0.08)$ \\
\hline$\left[\mathrm{La}^{-}\right]_{\text {peak }}\left(\mathrm{mmol} \cdot \mathrm{L}^{-1}\right)$ & $4.5 \pm 1.4$ & $6.2 \pm 2.4$ & $<0.01$ & $1.7[0.7,2.8] ; 38,0 \%$ & 0.90 , Moderate & $-0.52(0.05)$ \\
\hline HR (bpm) & $185 \pm 8$ & $184 \pm 7$ & - & - & - & $-0.47(0.09)$ \\
\hline Aer energy (kJ) & $287 \pm 57$ & $281 \pm 57$ & - & - & - & $-0.21(0.48)$ \\
\hline AnL energy $(\mathrm{kJ})$ & $10.75 \pm 5.55$ & $16.70 \pm 9.26$ & $<0.01$ & $5.95[2.39,9.51] ; 55.4 \%$ & 0.92 , Moderate & $-0.48(0.08)$ \\
\hline AnAL energy $(k J)$ & $24.82 \pm 4.41$ & $25.45 \pm 4.38$ & $<0.01$ & $0.63[0.27,0.98] ; 2.5 \%$ & 0.99, Moderate & $-0.34(0.23)$ \\
\hline Aer contribution (\%) & $88.90 \pm 2.46$ & $87.06 \pm 1.92$ & $<0.01$ & $-1.83[-2.69,-0.98] ;-2.1 \%$ & -1.19, Moderate & $0.41(0.14)$ \\
\hline AnL contribution (\%) & $3.32 \pm 1.73$ & $4.96 \pm 2.13$ & $<0.01$ & $1.63[0.79,2.47] ; 49.4 \%$ & 1.08, Moderate & $-0.50(0.07)$ \\
\hline AnAL contribution (\%) & $7.78 \pm 1.06$ & $7.98 \pm 1.06$ & - & - & - & $0.18(0.54)$ \\
\hline $\mathrm{E}_{\mathrm{tot}}(\mathrm{kJ})$ & $322.3 \pm 57.0$ & $324.0 \pm 66.7$ & - & - & - & $-0.27(0.34)$ \\
\hline$\dot{\mathrm{E}}(\mathrm{kW})$ & $0.90 \pm 0.16$ & $0.94 \pm 0.21$ & - & - & - & $0.08(0.79)$ \\
\hline$C\left(\mathrm{~kJ} \cdot \mathrm{m}^{-1}\right)$ & $0.80 \pm 0.14$ & $0.81 \pm 0.17$ & - & - & - & $-0.28(0.34)$ \\
\hline
\end{tabular}

MRT: mean response time; $\mathrm{A}_{\mathrm{p}}$ : amplitude of the fast $\mathrm{VO}_{2}$ component; $\mathrm{VO}_{2}$ : oxygen uptake; [La $\left.{ }^{-}\right]_{\text {peak: }}$ peak blood lactate; HR: heart rate; Aer: aerobic; AnL: anaerobic lactic; AnAL: anaerobic alactic; $E_{\text {tot }}$ : total energy expenditure; $\dot{E}:$ metabolic power; $C$ : energy cost; -: Not significantly different.

POST associated with a slower performance time $(p \leq 0.02)$. Changes in SL at 100-m, v, and v at 200-, 300- and 400-m were also highly correlated with the reduction in swimming performance (good to excellent; $p \leq 0.03$; Table 2 ).

The $A_{p}$ ( $p=0.11$; mean difference: $-3.4,95 \% \mathrm{Cl}:-7.8$ to $\left.-0.9 \mathrm{~mL} \cdot \mathrm{kg}^{-1} \cdot \mathrm{min}^{-1} ; d=-0.44\right)$ and MRT ( $\mathrm{p}=0.36 ; 3.6,-4.5$ to $11.6 \mathrm{~s} ; d=0.25$ ) were similar between PRE and POST conditions and both parameter estimates had a coefficient of variation of $1 \%$ ). $\mathrm{VO}_{2}$ POST was lower at both the 100 ( $\mathrm{p} \leq 0.010$; $d=-1.26)$ and 200-m intervals ( $\mathrm{p}=0.02 ; d=-0.66)$, but similar at the $300-\mathrm{m}$ lap $\left(\mathrm{p}=0.07 ;-3.9,-8.2\right.$ to $0.4 \mathrm{~mL} \cdot \mathrm{kg}^{-1} \cdot \mathrm{min}^{-1}$; $d=-0.50)$ and at the $\mathrm{T} 400$ end $\left(\mathrm{VO}_{2 \text { max }} ; \mathrm{p}=0.10 ;-3.1,-7.0\right.$ to $0.7 \mathrm{~mL} \cdot \mathrm{kg}^{-1} \cdot \mathrm{min}^{-1} ; d=-0.45$; Table 3). The $\left[\mathrm{La}^{-}\right]_{\text {peak }}$ at POST was higher than PRE condition $(p<0.01 ; d=0.90)$ and heart rate was similar between testing sessions $(p=0.49 ;-1,-5$ to 2 bpm; $d=-0.18$ ). The Aer energy pathway ( $\mathrm{p}=0.64 ;-6 ;-31$ to $20 \mathrm{~kJ} ; d=-0.12$ ) was similar between conditions, but AnL POST ( $<<0.01 ; d=0.92$ ) and AnAL POST ( $p<0.01 ; d=0.99$ ) were higher (Table 3 ). However, Aer contribution at POST was $\sim 1.8 \%$ lower $(95 \% \mathrm{Cl}:-2.69$ to $-0.98 \% ; \mathrm{p}<0.01 ; d=-1.19)$ and AnL contribution $\sim 1.6 \%$ higher $(0.8$ to $2.5 \%$; $<<0.01 ; d=1.08$; Table 3), despite a similar AnAL contribution ( $p=0.55 ; 0.20$, -0.49 to $-0.90 \% ; d=0.16)$. The $\mathrm{E}_{\mathrm{tot}}(\mathrm{p}=0.89 ; 1.7,-25.8$ to $29.3 \mathrm{~kJ} ; d=0.03), \dot{\mathrm{E}}(\mathrm{p}=0.25 ; 0.04,-0.03$ to $0.12 \mathrm{~kW} ; d=0.30$ ) and $C\left(p=0.92 ; 0.01,-0.06\right.$ to $0.07 \mathrm{~kJ} \cdot \mathrm{m}^{-1} ; d=0.02$ ) were similar between PRE and POST. A good correlation was evident between changes in $\left[\mathrm{La}^{-}\right]_{\text {peak }}$ and swimming performance impairment $(r=-0.52 ; p=0.05$; Table 3$)$.

\section{Discussion}

Swimmers showed a substantial decrease in performance after four-weeks of training cessation, despite the sustained MRT, $A_{p}$ and $\dot{\mathrm{V}}_{2 \max }$ values. Although the Aer contribution decreased $\sim 1.8 \%$ and the AnL contribution increased $\sim 1.6 \%$, $E_{\text {tot }}$ and $C$ remained similar after the off-season. The impaired performance was mainly associated with lower SR (at all 100-m intervals), SL (only in the 100-m lap), [ $\left.\mathrm{La}^{-}\right]_{\text {peak }}$ and non-swimming specific physical activities conducted during the off-season. Although the height, arm span and weight values increased after four-weeks of training cessation, they did not significantly account for variance in performance decrements. However, impairment in swimming performance was attenuated in those swimmers who were more physically active during the detraining period.

Selected energetic and kinematic determinants of swimming performance start to decay when the training process is broken, leading to detraining and reduced performance (Billat et al., 1996; Mujika \& Padilla, 2000). Although PRE and POST $E_{\text {tot }}$ and $C$ values were similar, other energetic and kinematic markers contributed to maintenance of performance after the off-season. Although MRT, $A_{p}, \dot{V} \mathrm{O}_{2 \max }$ and Aer energy pathway were essentially unchanged over fourweeks of training cessation, the Aer contribution decreased by $\sim 1.8 \%$, particularly during the first $200-\mathrm{m}$ where $\dot{\mathrm{VO}}_{2}$ was lower. The AnL energetic contribution increased from PRE to POST, correlating negatively with T400 swimming performance (Table 3 ) increasing the contribution to $E_{\text {tot }}$ by $\sim 1.6 \%$. Although similar SL were exhibited in-between evaluations, these changes in energy contributions were associated with lower SR values. However, $E_{\text {tot }}$ and $C$ remained unchanged, and their trivial delta values were not correlated with change in swimming time-trial performance. Thus, energy pathways and respective contributions were more sensitive variables than $E_{\text {tot }}$ and $C$ to identify the modest changes in energetics after the off-season. It seems that a lower aerobic metabolism efficiency and a higher anaerobic contribution make it harder for a swimmer to sustain the $v$ reached prior to the off-season period. Perhaps, changes in muscle structure during cessation are explained by a decrease in both mitochondrial content and mitochondrial function, thus impairing Aer energy pathway (Bishop, Granata, \& Eynon, 2014; Granata, Oliveira, Little, Renner, \& Bishop, 2016).

The T400 was chosen for the present study given its validity and feasibility for assessing aerobic power in age-group 
swimmers (Billat et al., 1996; Lavoie, Leger, Montpetit, \& Chabot, 1983; Reis, Alves, Bruno, Vleck, \& Millet, 2012; Zacca et al., 2017). In fact, high correlations for $\mathrm{HR}$ (also for $\dot{\mathrm{VO}}_{2 \max }$ and ventilation) and high reproducibility for all primary energetic variables indicated a close agreement between the seventh step of the $7 \times 200-m$ incremental intermittent protocol (the standard protocol for swimming training diagnosis; Fernandes et al., 2003; Pyne, Lee, \& Swanwick, 2001) and the T400. It is also important to recognize a limitation of this study design. Although HR remained unchanged from PRE to POST, which could indicate that four-weeks are not sufficient for changes in myocardial structure, we did not collect any other cardiovascular variables.

The observed $E_{\text {tot }}$ and $C$ values are in line with the literature (Fernandes et al., 2006; Ribeiro et al., 2017; Sousa et al., 2013) and, although both are associated with swimming performance, they rely on a swimmer's $v$ to accomplish a certain distance. The $v$ provides overall information about a swimmer's status, with its kinetics a product of the underlying technical proficiency, reflecting the balance between propulsive and drag forces (related to motor control). The $v$ was lower after four-weeks of training cessation regardless the absence of a marked difference in $\mathrm{VO}_{2 \max }$. This $\mathrm{v}$, assumed to correspond with the $\mathrm{vVO}_{2 \max }$, distinguishes individual differences in performance that $\dot{V}_{2 \max }$ or $\mathrm{C}$ alone could not identify, with swimmers with similar $\dot{\mathrm{V}}_{2 \max }$ showing different performance times (Billat et al., 1996; Daniels, 2013; Zacca et al., 2017). In fact, changes in $\mathbf{v} \mathrm{VO}_{2 \max }$ were associated with individual differences in the T400 performance that $\dot{\mathrm{V}}_{2 \max }$ or $\mathrm{C}$ did not identify. Therefore, coaches and researchers should focus on $\mathrm{v}^{\mathrm{V}} \mathrm{O}_{2 \max }$ rather than $\dot{\mathrm{VO}}_{2}$ and/or $\mathrm{C}$ when evaluating energetics in age-group swimmers.

Regarding swimming technique, it is relevant to highlight the importance of the pull and push as the main propulsive phases in front crawl (Figueiredo et al., 2013; Seifert et al., 2014). Theoretically, a continuous swimming motor pattern can minimise the $C$ indicated by lower intra-cyclic speed variation (Figueiredo et al., 2013; Seifert et al., 2014). However, intra- and inter-individual adaptations occur when accomplishing a given task-goal, meaning that the coordination pattern variability leads to functional adaptations to constraints. However, even in the absence of an ideal coordination pattern that swimmers should replicate (Seifert et al., 2014), the IdC PRE and POST values reflected the same coordination mode (catch-up; IdC: PRE between $-9.3 \%$ and $-8.5 \%$; POST between $-10.1 \%$ and $-9.7 \%$ ) across all the T400 $100-\mathrm{m}$ intervals, mainly due to the event (400-m) and study design characteristics (POST condition at lower $v$ ) (Ribeiro et al., 2017). Swimming efficiency (SI) for all 100-m intervals decreased after the detraining period, as a function of lower SR (despite similar values of SL). However, SI changes were not correlated with swimming performance, probably because the SR and SL patterns were not identical for all swimmers.

Impaired performance was associated with lower SR (at all 100-m intervals) and SL (only in the 100-m lap). Since the product between SR and SL represents the swimming speed, it is plausible that the marked association observed for SR and SL to changes in T400 are a consequence of the direct relationship between these variables and swimming performance (Costill et al., 1985; Morais et al., 2014). Since performance was impaired after off-season, we expected a decrease in $\dot{E}$, an increase in the C or both (Zamparo et al., 2011). However, both paramaters were largely unchanged (Table 3), perhaps due to intrasubject/intersubject variability and the modest degree of reliability in agegroup testing protocols (Reis et al., 2012).

Although some marked fluctuations were observed for mean weekly training volume between weeks 12-14 of the season (Figure 1), it is important to highlight that the PRE test was conducted after the main competition, i.e. there were other regional swimming competitions during the macrocycle. The absence of a classical reduction in training volume during the taper is somewhat atypical, but all training was prescribed by the coach without any external involvement from the investigators of this study. Despite this pattern of training, the progression of training load over the last 16-weeks macrocycle immediately before the off-season was $\sim 10 \%$ week $^{-1}$, which confirms the specific recommendations (increasing load in increments of $\sim 5-10 \%$ week $^{-1}$ to prevent injuries made for athletes (Hellard et al., 2015; Vanrenterghem et al., 2017; Walsh et al., 2011). Our expectation that young adolescent swimmers growth during the off-season period and contributions of non-swimming physical activity might offset the detraining related to swimming capacities was partially supported by the current results, in line with previous reports (Ayabakan et al., 2006; Csajagi et al., 2015; Kavouras \& Troup, 1996). Likewise, even after $\sim 10$-weeks of training cessation, 12-13 years old swimmers improved swimming kinematics as a function of growth and maturation (Moreira et al., 2014). However, POST anthropometric variables related to growth did not account for variation in performance after the fourweeks of training cessation. The amount of physical activity performed in the transition period in-between training seasons accounted for $40 \%$ of the total performance variance, and the decrease in swimming performance was lower in more physically active swimmers. Thus, swimmers should be physically active (mainly at moderate and vigorous intensity) when enjoying the off-season to minimise any decline in swimming performance. Constraints of finding an adequate sample of age-group swimmers with over 5 years of competitive experience and homogeneous pubertal maturation leaded us to pool and analyse male and female swimmers as a single group. However, although this methodological limitation should be considered, preliminary data analysis with all variables showed no significant sex effect.

\section{Conclusion}

Detraining after four-weeks of pool-based training cessation can impair swimming performance at the start of the following training season in age-group swimmers, highlighting the importance of maintaining fitness levels during off-season. Swimming performance in the $400 \mathrm{~m}$ front crawl was reduced by $3.8 \%$ after four-weeks of training cessation in competitive 14-15 years old swimmers. Impaired performance was mainly associated with reductions in $\mathrm{SR}$, an increase in $\left[\mathrm{La}^{-}\right]_{\text {peak }}$ and limited non-swimming specific physical activities. Although four-weeks was not long enough to detect a growth effect on performance, impairment of T400 performance was 
attenuated by those swimmers who were more physically active during the off-season. Thus, age-group swimmers should be physically active when enjoying the off-season, undertaking mainly moderate and vigorous activities. For example, running or cycling for aerobic conditioning, and dryland workouts (such as dry land sports, cross-training or gym-based exercises) could be helpful in minimising reductions in swimming performance during the transition to the following competitive season. It is important to keep in mind that training/detraining and subsequent adaptations are dynamic processes. In this sense, pool-based test sets, such as the T400 applied in the current study, should be useful in regularly monitoring swimmers.

\section{Disclosure statement}

No potential conflict of interest was reported by the authors.

\section{ORCID}

Rodrigo Zacca (D) http://orcid.org/0000-0003-0494-0000 Argyris Toubekis (D) http://orcid.org/0000-0002-2040-354X Laura Freitas (D) http://orcid.org/0000-0001-6758-4520 Ana Filipa Silva (D) http://orcid.org/0000-0002-1772-1272 Rui Azevedo (D) http://orcid.org/0000-0002-8904-002X João Paulo Vilas-Boas (D) http://orcid.org/0000-0002-4109-2939 David B. Pyne (D) http://orcid.org/0000-0003-1555-5079 Flávio A. De S. Castro (1D) http://orcid.org/0000-0003-0848-8226 Ricardo J. Fernandes (iD) http://orcid.org/0000-0002-5811-0443

\section{References}

Ayabakan, C., Akalin, F., Mengutay, S., Cotuk, B., Odabas, I., \& Ozuak, A. (2006). Athlete's heart in prepubertal male swimmers. Cardiology in the Young, 16(1), 61-66.

Barbosa, T. M., Bragada, J. A., Reis, V. M., Marinho, D. A., Carvalho, C., \& Silva, A. J. (2010). Energetics and biomechanics as determining factors of swimming performance: Updating the state of the art. Journal of Science and Medicine in Sport, 13(2), 262-269.

Barbosa, T. M., Morais, J. E., Marques, M. C., Silva, A. J., Marinho, D. A., \& Kee, Y. H. (2015). Hydrodynamic profile of young swimmers: Changes over a competitive season. Scandinavian Journal of Medicine \& Science in Sports, 25(2), e184-e196.

Billat, V., Faina, M., Sardella, F., Marini, C., Fanton, F., Lupo, S., ... Dalmonte, A. (1996). A comparison of time to exhaustion at VO2 max in elite cyclists, kayak paddlers, swimmers and runners. Ergonomics, 39(2), 267-277.

Binzoni, T., Ferretti, G., Schenker, K., \& Cerretelli, P. (1992). Phosphocreatine hydrolysis by 31P-NMR at the onset of constant-load exercise in humans. Journal of Applied Physiology, 73(4), 1644-1649.

Bishop, D. J., Granata, C., \& Eynon, N. (2014). Can we optimise the exercise training prescription to maximise improvements in mitochondria function and content? Biochimica et biophysica acta, 1840(4), 1266-1275.

Chollet, D., Chalies, S., \& Chatard, J. C. (2000). A new index of coordination for the crawl: Description and usefulness. International Journal of Sports Medicine, 21(1), 54-59.

Costill, D. L., Fink, W. J., Hargreaves, M., King, D. S., Thomas, R., \& Fielding, R. (1985). Metabolic characteristics of skeletal muscle during detraining from competitive swimming. Medicine and Science in Sports and Exercise, 17(3), 339-343.

Costill, D. L., Kovaleski, J., Porter, D., Kirwan, J., Fielding, R., \& King, D. (1985). Energy expenditure during front crawl swimming: Predicting success in middle-distance events. International Journal of Sports Medicine, 6(5), 266-270.

Craig, C. L., Marshall, A. L., Sjostrom, M., Bauman, A. E., Booth, M. L., Ainsworth, B. E., ... Oja, P. (2003). International physical activity questionnaire: 12 -country reliability and validity. Medicine and Science in Sports and Exercise, 35(8), 1381-1395.

Csajagi, E., Szauder, I., Major, Z., \& Pavlik, G. (2015). Left ventricular morphology in different periods of the training season in elite young swimmers. Pediatric Exercise Science, 27(2), 185-191.

Daniels, J. (2013). Daniels' running formula. Human kinetics Publisher; 3rd Edition. In Champaign, IL; ISBN-13 (pp. 9781450431835).

de Jesus, K., Guidetti, L., de Jesus, K., Vilas-Boas, J. P., Baldari, C., \& Fernandes, R. J. (2014). Which are the best VO2 sampling intervals to characterize low to severe swimming intensities? International Journal of Sports Medicine, 35(12), 1030-1036.

Ferguson, C. J. (2009). An effect size primer: A guide for clinicians and researchers. Professional Psychology: Research and Practice, 40(5), 532-538.

Fernandes, R. J., Billat, V. L., Cruz, A. C., Colaco, P. J., Cardoso, C. S., \& VilasBoas, J. P. (2006). Does net energy cost of swimming affect time to exhaustion at the individual's maximal oxygen consumption velocity? The Journal of Sports Medicine and Physical Fitness, 46(3), 373-380.

Fernandes, R. J., Cardoso, C. S., Soares, S. M., Ascensao, A., Colaco, P. J., \& Vilas-Boas, J. P. (2003). Time limit and VO2 slow component at intensities corresponding to VO2max in swimmers. International Journal of Sports Medicine, 24(8), 576-581.

Figueiredo, P., Pendergast, D. R., Vilas-Boas, J. P., \& Fernandes, R. J. (2013). Interplay of biomechanical, energetic, coordinative, and muscular factors in a $200 \mathrm{~m}$ front crawl swim. BioMed Research International, (2013, 897232.

Figueiredo, P., Toussaint, H. M., Vilas-Boas, J. P., \& Fernandes, R. J. (2013). Relation between efficiency and energy cost with coordination in aquatic locomotion. European Journal of Applied Physiology, 113(3), 651-659.

Fleiss, J. (1986). The design and analysis of clinical experiments. New York, NY: Wiley.

Garrido, N., Marinho, D. A., Reis, V. M., van Den Tillaar, R., Costa, A. M., Silva, A. J., \& Marques, M. C. (2010). Does combined dry land strength and aerobic training inhibit performance of young competitive swimmers? Journal of Sports Science \& Medicine, 9(2), 300-310.

Granata, C., Oliveira, R. S., Little, J. P., Renner, K., \& Bishop, D. J. (2016). Mitochondrial adaptations to high-volume exercise training are rapidly reversed after a reduction in training volume in human skeletal muscle. FASEB Journal, 30(10), 3413-3423.

Hanon, C., Leveque, J. M., Thomas, C., \& Vivier, L. (2008). Pacing strategy and VO2 kinetics during a 1500-m race. International Journal of Sports Medicine, 29(3), 206-211.

Hellard, P., Avalos, M., Guimaraes, F., Toussaint, J. F., \& Pyne, D. B. (2015). Training-related risk of common illnesses in elite swimmers over a 4-yr period. Medicine and Science in Sports and Exercise, 47(4), 698-707.

Hopkins, W. G. (2002) A scale of magnitudes for effect statistics. A new view of statistics. Retrieved from http://sportsci.org/resource/stats/ effectmag.html

Ingham, S. A., Fudge, B. W., Pringle, J. S., \& Jones, A. M. (2013). Improvement of $800-\mathrm{m}$ running performance with prior high-intensity exercise. International Journal of Sports Physiology and Performance, 8 (1), 77-83.

Kavouras, S., \& Troup, J. (1996). Growth and developmental changes in selected characteristics of elite age group swimmers. In J. P. Troup, A. P. Hollander, D. Strasse, S. W. Trappe, J. M. Cappaert, \& T. A. Trappe (Eds.), BMS VII. Proceedings of the biomechanics and medicine in swimming VII (pp. 234-240). London: E \& FN Spon.

Laffite, L. P., Vilas-Boas, J. P., Demarle, A., Silva, J., Fernandes, R., \& Billat, V. L. (2004). Changes in physiological and stroke parameters during a maximal 400-m free swimming test in elite swimmers. Canadian Journal of Applied Physiology, 29(Suppl), S17-S31.

Lavoie, J. M., Leger, L. A., Montpetit, R. R., \& Chabot, S. (1983). Backward extrapolation of $\mathrm{VO} 2$ from the $\mathrm{O} 2$ recovery curve after a voluntary maximal $400 \mathrm{~m}$ swim. In A. P. Hollander, P. A. Huijing, \& G. De Groot (Eds.), Biomechanics and medicine in swimming (pp. 222-227). Champaign, IL: Human Kinetics Publishers.

Ma, S., Rossiter, H. B., Barstow, T. J., Casaburi, R., \& Porszasz, J. (2010). Clarifying the equation for modeling of $\mathrm{VO} 2$ kinetics above the lactate threshold. Journal of Applied Physiology, 109(4), 1283-1284. 
Morais, J. E., Marques, M. C., Marinho, D. A., Silva, A. J., \& Barbosa, T. M. (2014). Longitudinal modeling in sports: Young swimmers' performance and biomechanics profile. Human Movement Science, 37, 111-122.

Moreira, M. F., Morais, J. E., Marinho, D. A., Silva, A. J., Barbosa, T. M., \& Costa, M. J. (2014). Growth influences biomechanical profile of talented swimmers during the summer break. Sports Biomechanics, 13(1), 62-74.

Mujika, I., Busso, T., Lacoste, L., Barale, F., Geyssant, A., \& Chatard, J. C. (1996). Modeled responses to training and taper in competitive swimmers. Medicine and Science in Sports and Exercise, 28(2), 251-258.

Mujika, I., Chatard, J. C., Busso, T., Geyssant, A., Barale, F., \& Lacoste, L. (1995). Effects of training on performance in competitive swimming. Canadian Journal of Applied Physiology, 20(4), 395-406.

Mujika, I., \& Padilla, S. (2000). Detraining: Loss of training-induced physiological and performance adaptations. Part I: Short term insufficient training stimulus. Sports Medicine, 30(2), 79-87.

Neufer, P. D. (1989). The effect of detraining and reduced training on the physiological adaptations to aerobic exercise training. Sports Medicine, 8 (5), 302-320.

Pyne, D. B., Lee, H., \& Swanwick, K. M. (2001). Monitoring the lactate threshold in world-ranked swimmers. Medicine and Science in Sports and Exercise, 33(2), 291-297.

Reis, J. F., Alves, F. B., Bruno, P. M., Vleck, V., \& Millet, G. P. (2012). Oxygen uptake kinetics and middle distance swimming performance. Journal of Science and Medicine in Sport, 15(1), 58-63.

Ribeiro, J., Figueiredo, P., Guidetti, L., Alves, F., Toussaint, H., Vilas-Boas, J. P., ... Fernandes, R. J. (2016). AquaTrainer ${ }^{\circledast}$ snorkel does not increase hydrodynamic drag but influences turning time. International Journal of Sports Medicine, 37(4), 324-328.

Ribeiro, J., Figueiredo, P., Morais, S., Alves, F., Toussaint, H., Vilas-Boas, J. P., \& Fernandes, R. J. (2017). Biomechanics, energetics and coordination during extreme swimming intensity: Effect of performance level. Journal of Sports Sciences, 35(16), 1614-1621.

Ribeiro, J., Toubekis, A. G., Figueiredo, P., de Jesus, K., Toussaint, H. M., Alves, F., ... Fernandes, R. J. (2017). Biophysical determinants of front-crawl swimming at moderate and severe intensities. International Journal of Sports Physiology and Performance, 12(2), 241-246.
Seifert, L., Komar, J., Barbosa, T., Toussaint, H., Millet, G., \& Davids, K. (2014). Coordination pattern variability provides functional adaptations to constraints in swimming performance. Sports Medicine, 44 (10), 1333-1345.

Seiler, S. (2010). What is best practice for training intensity and duration distribution in endurance athletes? International Journal of Sports Physiology and Performance, 5(3), 276-291.

Sousa, A., Figueiredo, P., Zamparo, P., Vilas-Boas, J. P., \& Fernandes, R. J. (2013). Anaerobic alactic energy assessment in middle distance swimming. European Journal of Applied Physiology, 113(8), 2153-2158.

Sousa, A. C., Vilas-Boas, J. P., \& Fernandes, R. J. (2014). VO2 kinetics and metabolic contributions whilst swimming at 95,100 , and $105 \%$ of the velocity at VO2max. BioMed Research International, (2014, 675363.

Tanner, J. M., \& Whitehouse, R. H. (1976). Clinical longitudinal standards for height, weight, height velocity, weight velocity, and stages of puberty. Archives of Disease in Childhood, 51(3), 170-179.

Thevelein, X., Daly, D., \& Persyn, U. (1984). Measurement of total energy use in the evaluation of competitive swimmers. In N. Bachl, L. Prakop, \& R. Suckaert (Eds.), Current topics in sports medicine (pp. 668-676). Wien: Urban \& Schwartzenberg.

Tschiene, P. (1977). Einige neue Aspekte zur Periodisierung des Hochleistungstrainings. Leistungssport, 5, 379-382.

Vanrenterghem, J., Nedergaard, N. J., Robinson, M. A., \& Drust, B. (2017). Training load monitoring in team sports: A novel framework separating physiological and biomechanical load-adaptation pathways. Sports Medicine, 47(11), 2135-2142.

Walsh, N. P., Gleeson, M., Pyne, D. B., Nieman, D. C., Dhabhar, F. S., Shephard, R. J., ... Kajeniene, A. (2011). Position statement. Part two: Maintaining immune health. Exercise Immunology Review, 17, 64-103.

Zacca, R., Azevedo, R., Silveira, R. P., Vilas-Boas, J. P., Pyne, D. B., de S Castro, F. A., \& Fernandes, R. J. (2017). Comparison of incremental intermittent and time trial testing in age-group swimmers. Journal of Strength and Conditioning Research. doi:10.1519/jsc.0000000000002087

Zamparo, P., Capelli, C., \& Pendergast, D. (2011). Energetics of swimming: A historical perspective. European Journal of Applied Physiology, 111(3), 367-378. 


\section{Effects of detraining in age-group swimmers performance, energetics and kinematics}

Rodrigo Zacca, Argyris Toubekis, Laura Freitas, Ana Filipa Silva, Rui Azevedo, João Paulo Vilas-Boas, David B. Pyne, Flávio A. De S. Castro \& Ricardo J. Fernandes

To cite this article: Rodrigo Zacca, Argyris Toubekis, Laura Freitas, Ana Filipa Silva, Rui Azevedo, João Paulo Vilas-Boas, David B. Pyne, Flávio A. De S. Castro \& Ricardo J. Fernandes (2019): Effects of detraining in age-group swimmers performance, energetics and kinematics, Journal of Sports Sciences, DOI: 10.1080/02640414.2019.1572434

To link to this article: https://doi.org/10.1080/02640414.2019.1572434

Published online: 04 Feb 2019.

Submit your article to this journal \lceil

View Crossmark data ¿ 